\title{
Effects of the Counter Ion Valency on the Colloidal Interaction between Two Cylindrical Particles
}

\author{
Inho Lee, ${ }^{\dagger \neq}$ Hyunbae Dong, ${ }^{\dagger \neq}$ Juyoung Choi, ${ }^{\dagger}$ and Sang-Yup Lee ${ }^{\dagger .^{-}}$ \\ - Department of Chemical and Biomolectlar Engineering, ${ }^{\ddagger}$ Specialized Graduate School of Hydrogen \& Fuel Cell, \\ Yonse Universitw; Seoul 120-749. Korea. E-mail: leessyovvonsei.ac.hr \\ Received Juty 29, 2008, Accepted December 10, 2008
}

\begin{abstract}
In this study, the effects of counter ion valency of the electrolyte on the colloidal repulsion between two parallel cylindrical particles were investigated. Electrostatic interactions of the cylindrical particles were calculated with the variation of counter ion valency. To calculate the electrical repulsive energy working between these two cylindrical particles, Derjaguin approximation was applied. The electrostatic potential profiles were obtained numerically by solving nonlinear Poission-Boltzmann (P-B) equation and calculating middle point potential and repulsive energy: working between interacting surfaces. The electrical potential and repulsive energy were influenced by counter ion valency, Debye length, and surface potential. The potential profile and middle point potential decayed with the counter ion valency due to the promoted shielding of electrical charge. On the while, the repulsive energy increased with the counter ion valency at a short separation distance. These behaviors of electrostatic interaction agreed with previous results on planar or spherical surfaces.
\end{abstract}

Key Words: Electrical repulsion. Derjaguin approximation. Ion valency. Cỵlinder

\section{Introduction}

Colloidal suspension containing cylindrical particles such as nanotube and nanorod has been studied widely for their promising applications in engineering. ${ }^{1 \cdot 3}$ Various methods such as soft and hard templating methods and controlled epitaxial grow th have been developed to synthesize nanorods and nanotubes. ${ }^{4 \cdot ?}$ However. not only the synthesis but the manipulation of synthesized nanorods and nanotubes are required for the practical application. For the manipulation of these cylindrical particles. the analysis on the interaction between these particles is required. which has been of keen interest in colloid science. ${ }^{8}$ Due to the anisotropy of cylindrical structure the analysis on the interaction between cylindrical particles is a complicate problem comparing to that of the isotropic particles like spheres. The colloidal interaction is dependent on various parameters such as ion concentration. surface potential and the valency of ions dissolved in aqueous medium. Specifically. the ion valency makes the interaction analysis more complicate because it would change the Debye length and make Poisson-Boltzmann (P-B) equation complex. In many previous studies. the P-B equation was solved for the case of monovalent (1:1) electrolyte: i.e. an electroly te composed of monovalent cation and anion, like $\mathrm{NaCl}$, is assumed to be dissolved in the suspending medium. When the colloidal system is assumed to have monovalent electrolyte, the P-B equation is expressed as a nonlinear equation containing hyperbolic sine function which can be simplified further after applying Debye-Hückel approximation. ${ }^{9}$ However, the P-B equation cannot be simply expressed in a hyperbolic function when divalent or trivalent electrolyte is used. Furthermore, linearization of $\mathrm{P}-\mathrm{B}$ equation would not be valid any more in a colloidal system with multivalent electroly te. ${ }^{[\hat{j} .11}$ Considering that many divalent or trivalent electrolytes are used as additives in preparing stable colloidal nanorod or nanotube suspension, it is required to study on the effects of ion valency on the colloidal repulsion between cylindrical particles.

Analy ses on the electrostatic repulsion between cylindrical particles have been studied analytically and numerically during last decades. Colloidal repulsion between cylindrical particles is largely dependent on the orientation of particles. From the previous studies. the electrical repulsion is nunimized when the particles are aligned crossed with each other because of the curvatures of interacting cylindrical surfaces. ${ }^{12,13}$ The electrical repulsion becomes larger with increase of particle length when the cylindrical particles are aligned parallel because of increase of interacting surface area. Therefore. the repulsion between cylindrical particles aligned in parallel is most important conparing to other configurations when considering the practical colloidal cylindrical particles with high aspect ratio

To analyze the repulsion between cylindrical particles aligned in parallel many assumptions were introduced. Firstly. the end effect was neglected. ${ }^{4-17}$ To solve the electrostatic repulsion analytically the surface potential was assumed to be lower than $25 \mathrm{mV}$ (at $25^{\circ} \mathrm{C}$ ) and the Debye screening length was much smaller than the radius of cylindrical particles. Under these assumptions, P-B equation was linearized and could be solved analytically. After obtaining the potential profile from the linearized equation, the interaction between cylindrical particles was obtained using superposition method. ${ }^{18}$ However. these analytical results are valid only when the colloidal particles are suspending in a concentrated ionic solution with low surface potential. Later. the low surface potential assumption was moderated by numerical technique. ${ }^{15.16,19-22}$ Using the numerical teclinique. more complicate problems such as overlapping of Debye screening length was solved. For example. electrical repulsive force of cylindrical particles confined in a rectangular channel was 
studied $^{15}$ and interaction free energy of two cylindrical particles was calculated using bycylinder coordinates. ${ }^{16}$ However. these numerical solutions were performed only for the cases of symmetric monovalent electrolytes. For the effect of multivalent ions on the colloidal interaction. only several studies have been carried out. A simplified analytic solution of P-B equation in 2:1 electrolyte was reported ${ }^{-3}$ and the electrostatic force working between spheres was recently analy $\angle$ ed. ${ }^{11}$

In this work. the electrostatic repulsive energies between two cylindrical particles were investigated by varying the ion valency of unsy'mmetrical electrolyte. Specifically the effects of the counter ion valency to the cylindrical particle surface were observed. Two cylindrical particles aligned in parallel are modeled and the electrostatic repulsive energy between them is investigated. The electrostatic repulsion is important specifically when these particles are positioned close where the electrical double layers (or Debye lengths) near the particle surface are overlapped. Here. Derjaguin approximation is applied in calculating the interaction energy between cylindrical particles. To calculate the electrostatic repulsive energy the electrical potential profile is obtained by solving nonlinear $\mathrm{P}$-B equation, and then the osmotic pressure between interacting surface elements is calculated from the potential profile. This numerical approach would expand the insight on the colloidal interaction with complex situation.

\section{Formulation of the Problem}

Before the formulation of the problem. the following assumptions should be set up. Firstly. the cylinders were assumed to be charged negatively with constant surface potential. The use of a fixed surface potential as a boundary condition is more practical comparing to the use of surface charge density. The surface potential of the particle is estimated from the zeta potential measurement. Constant surface potential is known to be valid when the charge of colloidal particle is driven by the adsorption of ions. ${ }^{24}$ Secondly. electrostatic interaction is considered between only two cylinders of interest: the influence by other particles would be neglected. This assumption is valid for a dilute colloidal suspension under a certain critical concentration. ${ }^{35}$ Thirdly, the end effect of the cylindrical particle is not considered. End effect is caused by the charge density different which is dependent to the curvature of the surface of interest. Finally the dielectric constant of the cylindrical particle is assumed to be zero to ignore the potential distribution inside of the cylindrical particle.

To formulate the electrostatic interaction between cylindrical particles. Derjaguin integration method was applied. Application of Derjaguin integration method is appropriate in a practical colloidal system where Debye length is smaller than or comparable to the radius of a cylinder. ${ }^{8}$ In practice. the Debye length of a solution containing $1 \mathrm{mM}$ of monovalent electrolyte is $9.6 \mathrm{~nm}$ (at $25^{\circ} \mathrm{C}$ ) and it becomes smaller as the ionic concentration increases. In contrast. the radius of cylindrical colloidal particle is larger than or comparable to the Deby'e screening length (for example. the radius of carbon nanotube is tens to hundreds $\mathrm{nm}$ ). Therefore, the Derjaguin approximation would be appropriate to model the practical system. The small Debye length suggests that the overlapping of electrical double layer would take place only when the separation distance is much smaller than the radius of cylindrical particles. Thereby, the electrical repulsion would be important only when the separation distance of two interacting surfaces is small enough.

To apply Derjaguin integration method. the interacting cylindrical surfaces are allocated to discrete interacting surface elements. Scheme of the interacting surface elements of model cylindrical particles is present in Figure 1 . The interacting surfaces are present as shadowed areas. Two cylinders in a length of $L$ and with a radius of $a$ are separated with a shortest distance of $H$. The surface potential of each cylinder is $\psi_{0}$. Between these two cylinders, the total interacting surface area is defined as the summation of planar rectangular elements which locates at the angle of $\theta_{i}$. The surface area of this element. $\Delta t$, is expressed as follows:

$$
\Delta A=a L\left(\sin \theta_{i}-\sin \theta_{i-1}\right)
$$

Exactly same surface area of interacting surface is applied for the pairing cylindrical particle. The discrete interacting surfaces are separated with a distance of $d$ that can be expressed as

$$
d=H+2 a\left(1-\cos \theta_{1}\right)
$$

Once the interacting surfaces are determined. the electrical potential profile between these surfaces and electrical potential at the middle point should be deternined. The electrical potential profile can be obtained by solving the nonlinear P-B equation. The $\mathrm{P}-\mathrm{B}$ equation is

$$
\nabla^{2} \psi=-\frac{1}{\varepsilon_{n} \varepsilon_{\mathrm{r}}} \sum F_{a} z C_{\alpha} \exp \left(-\frac{F_{a} z \psi}{R T}\right)
$$

where. $\varepsilon_{0}$ is vacuum permittivity $\varepsilon_{r}$ is relative permittivity of suspending medium, $F_{a}$ is Faraday constant. $z$ is ion valency, and $C_{\%}$ is bulk ion concentration in mole/L. With the

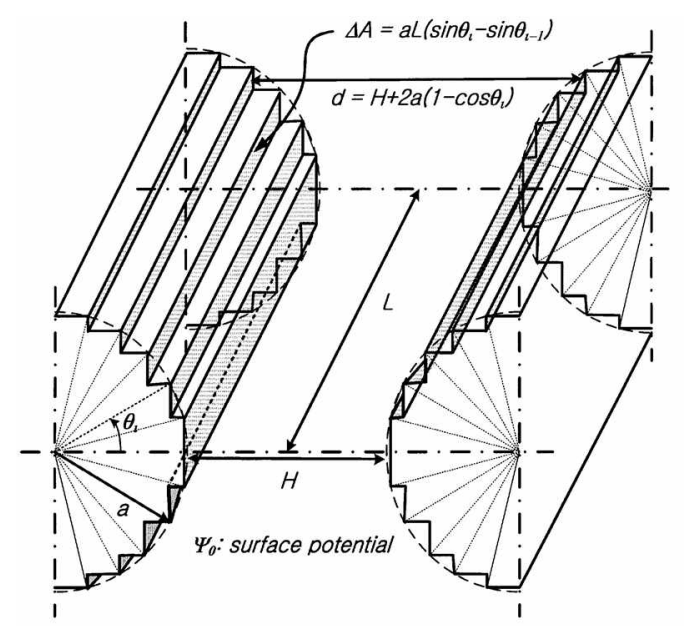

Figure 1. Scheme of the interacting surface elements (shadowed area) of cylinders 
variation of ion valency of $z$, equations of P-B equation. Debye screening length. pressure difference and the repulsive energy would change.

Imagine that the cylinder is charged negatively and an electrolyte composed of one cation whose valency is $z$ and monovalent anions. For example. $\mathrm{CaCl}_{2}$ and $\mathrm{AlCl}_{3}$ would have $z$ values of 2 and 3 , respectively. For the colloidal solution containing such a multivalent electrolyte. the P-B equation can be expressed as equation (4) after the consideration of Derjaguin approximation.

$$
\frac{d^{2} \psi}{d x^{2}}=\frac{2 z F_{a} C_{n}}{\varepsilon_{0} \varepsilon_{r}} \cdot \frac{1}{2}\left\{\exp \left(\frac{F_{a} \psi}{R T}\right)-\exp \left(-\frac{z F_{a} \psi}{R T}\right)\right\}
$$

By introducing dimensionless parameters present in equation (5). the $\mathrm{P}-\mathrm{B}$ equation is transformed to a dimensionless form of equation (6).

$$
\begin{aligned}
& \ddot{\psi}=\frac{F_{a} \psi}{R T} \text { and } y=\frac{x}{a} \\
& \frac{d^{\hat{1}} \tilde{\psi}}{d^{2}}=\frac{1}{2 z} \frac{a^{2}}{\lambda_{D}^{2}}\left(e^{\bar{\psi}}-e^{-z \bar{\psi}}\right)
\end{aligned}
$$

In equation (6), the Debye length. $\lambda_{D}$, is defined as

$$
\frac{1}{\lambda_{V}^{2}}=k^{2}=\frac{2 z^{2} F_{a}^{2} C_{z}}{\varepsilon_{0} \varepsilon_{r} R T}
$$

When solving above dimensionless P-B equation. the following boundary conditions are applied.

$$
\bar{\psi}=\bar{\psi}_{0}=\frac{F_{a} \psi_{0}}{R T} \text { at } y_{1}=0 \text { and } y_{2}=\frac{H+2 a\left(1-\cos \theta_{i}\right)}{a}
$$

Assigning the middle point potential as $\psi_{n}$. the pressure difference. $\Delta P$, between the middle point and bulk solution is expressed as equation (9).

$$
\Delta P=2 C_{n} R T\left\{\frac{z}{2} \exp \left(\frac{F_{a} \psi_{m}}{R T}\right)+\frac{1}{2} \exp \left(-\frac{F_{a} z \psi_{m}}{R T}\right)-\left(\frac{z+1}{2}\right)\right\}
$$

The Maxwell stress was not considered here because of the symmetric potential distribution between interacting surfaces. Finally. the electrostatic repulsive energy working between interacting surface elements. $\Delta r_{r, s}$ can be obtained by integrating above pressure difference from the dimensionless
Table 1. Variation of Debve lengths with the electrolyte concentration and ion valencies $\left(25^{\circ} \mathrm{C}\right)$

\begin{tabular}{crrr}
\hline Electrolyte concentration & \multicolumn{3}{c}{ Debye length [mm] } \\
\cline { 2 - 4 }$[\mathrm{m} M]$ & $\mathrm{Z}=1$ & $\mathrm{Z}=2$ & $\mathrm{Z}=3$ \\
\hline 0.1 & 30.39 & 15.20 & 10.13 \\
1 & 9.61 & 4.81 & 3.20 \\
10 & 3.04 & 1.52 & 1.01 \\
100 & 0.96 & 0.48 & 0.32 \\
\hline
\end{tabular}

separation distance $y_{2}$ to infinite.

$$
\begin{aligned}
\Delta r_{m_{1}}= & a^{2} L\left(\sin \theta_{1}-\sin \theta_{i-1}\right) \int_{y_{3}} 2 C R T\left\{\frac{z}{2} \exp \left(\tilde{\psi}_{m}\right)+\right. \\
& \left.\frac{1}{2} \exp \left(-z \tilde{\psi}_{m}\right)-\left(\frac{z+1}{2}\right)\right\} d y
\end{aligned}
$$

The total repulsive energy is obtained by summarizing all the repulsive energies working between interacting surface elements. Thereby, the total repulsive energy is expressed as the following equation (11).

$$
v_{*}=\sum y_{i}
$$

Above equations could be applied to describe the repulsive energy of a cylindrical colloid suspending in the multivalent electrolyte solution.

In carrying out numerical calculation. the electrical potential profile was obtained from the solution of nonlinear $P-B$ equation of (6) by applying Gelakin finite element method combined with Newton's method. Quadratic basis functions were used in the calculation. The potential profile was obtained when the potential values were converged under the tolerance of $1 \times 10^{-4}$. After obtaining the electrical potential profile. the electrical potential at the middle point. $\psi_{m}$, was obtained, and then the repulsive energy was calculated.

\section{Results and Discussion}

In general. Debye length decays with the electrolyte concentration. When nultivalent ions are dissolved in a colloidal solution. the Debye length would also reduce with increase of ion valency as indicated in equation (7). In Table 1, calculated (a)

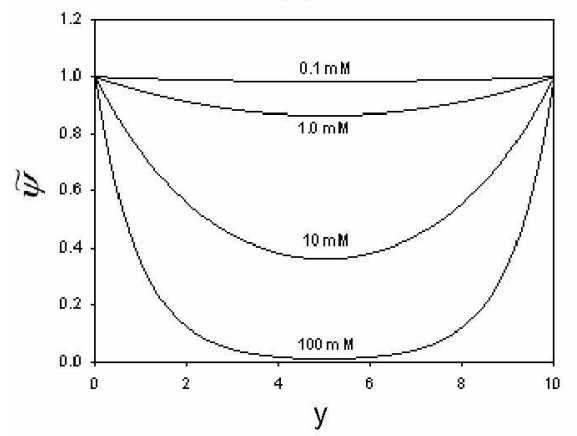

(b)

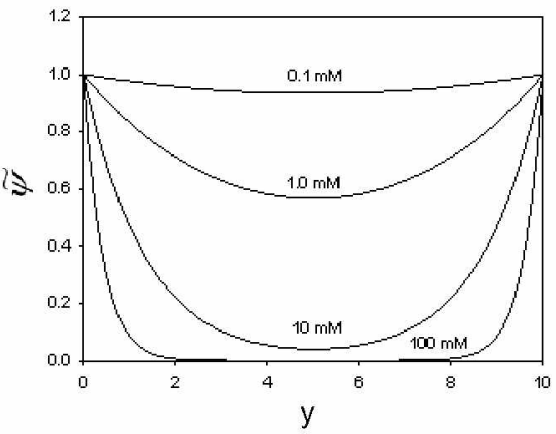

(c)

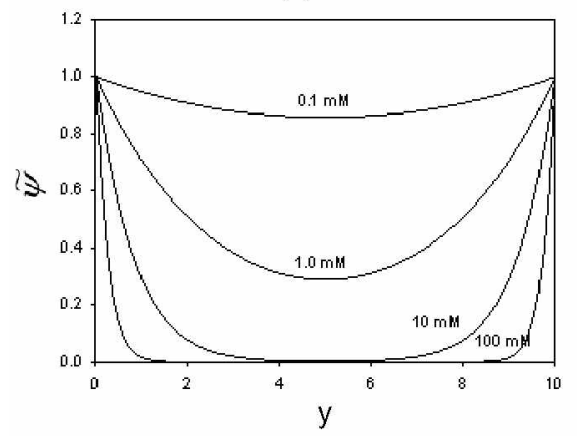

Figure 2. Electrical potential profile between two planar surfaces with the variation of ionic strength in (a) $1: 1$, (b) $2: 1$, and (c) $3: 1$ electrolytes. 
(a)

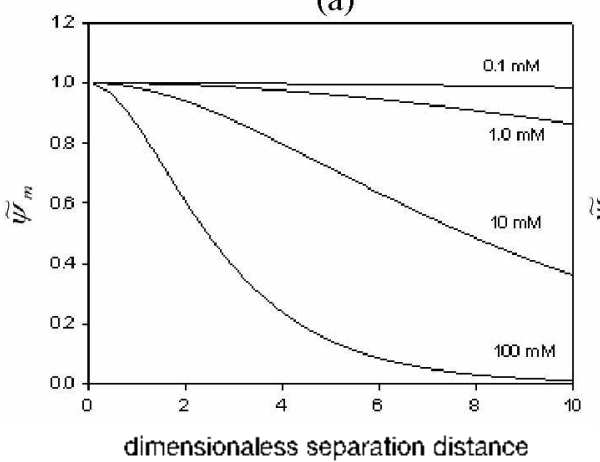

(b)

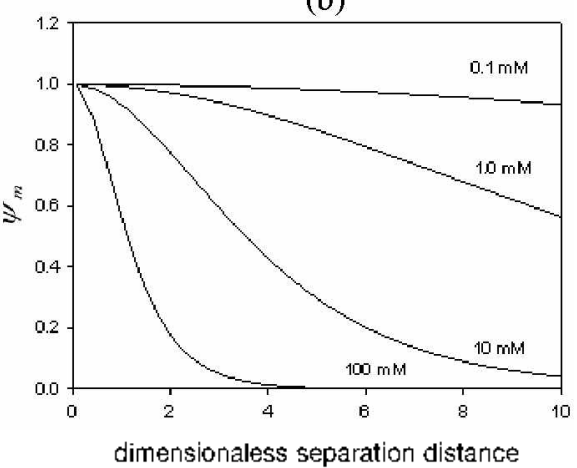

(c)

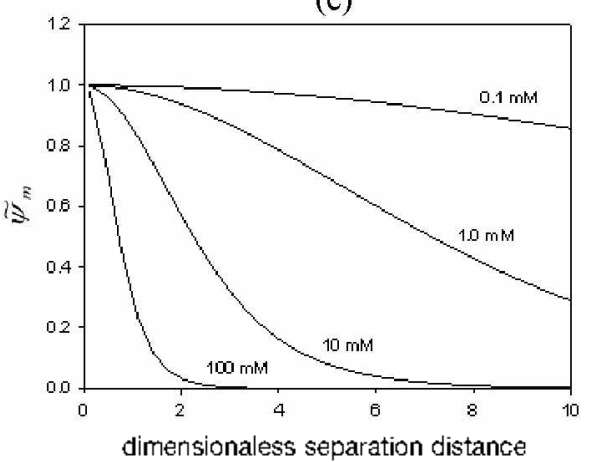

Figure 3. Profile of the middle point potential, $\ddot{\psi}_{m}$, with the variation of ionic strength in (a) $1: 1$, (b) $2: 1$, and (c) $3: 1$ electrolytes

Debye lengths at $25^{\circ} \mathrm{C}$ with the variation of electrolyte concentration and ion valency are present.

Before calculating the repulsive energies between cylindrical particles the electrical potential profiles were investigated. Electrical potential profiles between two parallel interacting surface elements were obtained from the solution of the dimensionless P-B equation of equation (6). The obtained electrical potential profile is present in Figure 2. In calculation the characteristic length of $a$ was arbitrarily chosen as $1 \mathrm{~nm}$, and the separation distance between surfaces was set as $10 \mathrm{~nm}$. Debye lengths at different ionic concentration are summarized in Table 1. Symmetric potential profiles between two surfaces were obtained under the assumption of the constant surface potential of $\widetilde{\psi}_{u}=1.0$. As present in Figure 1, the potential profile is symmetric since the surface potentials are same on both sides. Comparing figures $1(\mathrm{a}) \sim(\mathrm{c})$, the electrical potential decays more rapidly as the valency of cation increases when the ionic concentration is kept constant. Increase of counter ion valency reduced the Debye length and promoted the potential profile decay near the surface. The electrical potential also drops off faster with increase of electroly te concentration as it is known already. This electrical potential drop is generally explained by the screening of opposite charges and shrinkage of double layer thickness with the ionic concentration.

From the potential profile between two parallel surfaces. the middle point potential. $\psi_{n}$, could be determined. The middle point potential is the potential value at the center of the separation distance. For example, the potential value at $v=5.0$ would be $\psi_{m}$ in Figure 2 . As described in equations ( 9 ) and (10), determination of middle point potential is crucial in calculating repulsive energy. Since the middle point potential would change with the separation distances between two surfaces. it would decay as the separation distance becomes wider. In Figures 3 and 4 , changes of $\widetilde{\psi}_{m}$ are present. In Figure 3. profiles of $\widetilde{\psi}_{n}$ are present that were calculated at a constant surface potential of $\widetilde{\psi}_{n}=1.0$. At a weak ionic strength. the middle point potential changes little and whose value is close to surface potential. $\ddot{\psi}_{0}$. This little change indicates that the electrical potential profile extended long. which is representing as the thick Debye screening lengths. With increase of electrolyte concentration. $\widetilde{\psi}_{\mathrm{n} \text { i }}$ decays rapidly due to the screening effect. The middle point potential, $\tilde{\psi}_{n 1}$, decayed faster with increase of counter ion valency as presented in Figures $3(\mathrm{a}) \sim(\mathrm{c})$. This fast decay is driven by the promoted screening effect with the raise of counter ion valency.

Effects of surface potential $\widetilde{\psi}_{\diamond}$ on $\bar{\psi}_{m}$ were present in Figure 4. For the comparison. the nuiddle point potential was calculated at a constant ionic concentration of $100 \mathrm{mM}$. Increase of surface potential resulted in increases of $\widetilde{\psi}_{m}$. With increase of the separation distance. the $\widetilde{\psi}_{b i}$ decays. which represents electrical interaction is diminishing. The $\psi_{m}$ converged to zero at around the same separation distances regardless to the surface potential. This finding suggests that Debye length is the key factor deternining $\psi_{m}$ values rather than surface potential. Higher value of counter ion valency resulted in faster decay of $\widetilde{\psi}_{n}$ since the Debye length was reduced with the rise of ion valency. (a)

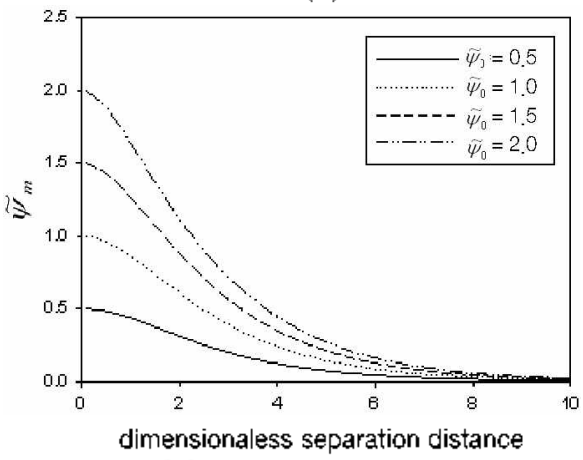

(b)

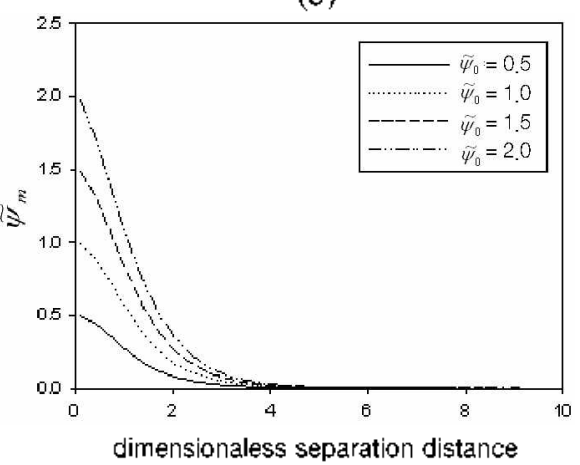

(c)

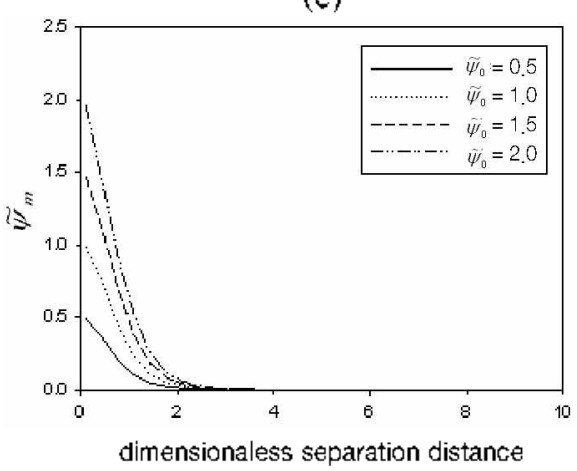

Figure 4. Profile of the middle point potential, $\ddot{\psi}_{m}$, with the variation of surface potential $\ddot{\psi}_{0}$ in (a) $1: 1$, (b) $2: 1$, and (c) $3: 1$ electrolytes. (Ionic strength is $100 \mathrm{mM}$ ) 

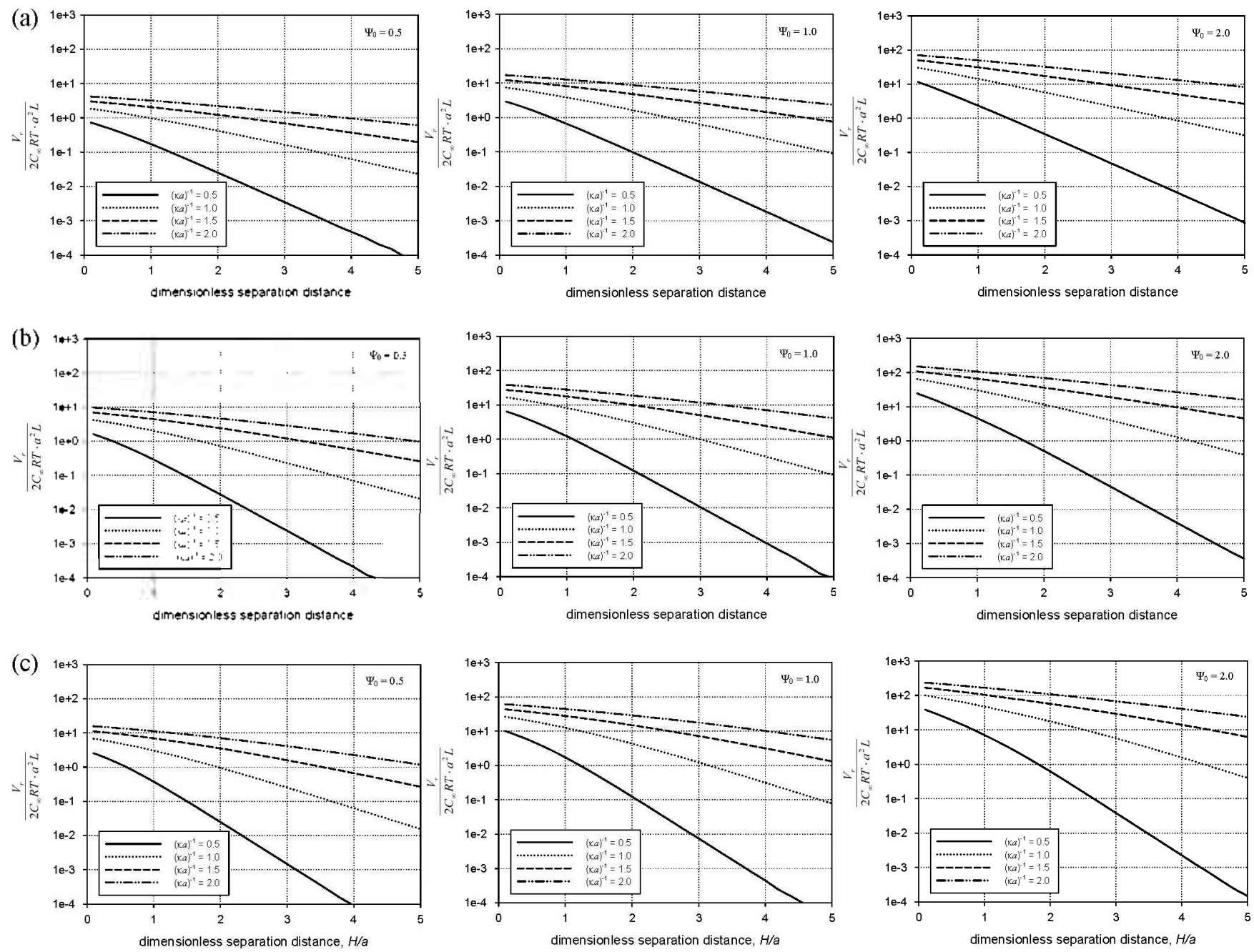

Figure 5. Electrical repulsive energy with different surface potential in (a) $1: 1$, (b) $2: 1$, and (c) $3: 1$ electrolytes.

After determination of the muddle point potential, $\widetilde{\psi}_{m}$. the electrical repulsive energy was calculated. As expressed in equation (10). the repulsive energy is dependent on both the counter ion valency. $z$. and the middle potential, $\breve{\psi}_{m}$. The dimensionless repulsive energy working between two prallel cylindrical particles. $\frac{\mathrm{I}}{2 C R T-\alpha^{2} L}$. is plotted against the dimensionless separa tion distance, H/a. For the comparison of ion valency effects, the dimensionless Debye length $\lambda_{D} / a$, and surface potentral. $\widetilde{\psi}_{v}$. were chosen as parameters in calculation. In Figure 5, the repulsive energy profiles against the separation distance. $\mathrm{Ha}$ are present. The repulsive energy is calculated at the same values of Debye length and surface potential. Regardless to the counter ion valency. several trends were commonly observed. Firstly, the repulsive energy increases with the Debye length. This Debye length dependency is reasonable considering that Debye length indicates the extent of the electrical double layer near to the surface." Increment of Debye length results in overlapping of the electrical double layers that leads to stronger repulsion. Secondly: surface potential increase led to higher repulsive energy. The repulsive energy is known to be proportional to the square of surface potential for the linearized P-B equation with $1: 1$ electrolyte. ${ }^{5}$ Our results showed similar dependency to the square of surface potential. but not exactly be proportional to it (data not shown). This difference is to be due to the nonlinearity of P-B equation used here and the curvature of the cylinder as discussed in the previous studies. $13.28,29$

Larger counter ion valency led to increment of repulsive energy. This repulsive energy raise by the counter ion valency agrees with previous results of the planar $r^{3}$ and spherical surfaces. ${ }^{3.1}$ The valency effect is donunant as the Debye length becomes thicker. Specifically. the repulsive energy of larger valency was lugher at a short separation distance, but the magnitude of repulsive energy turned over as the separation distance increases. This turnover of repulsive energy agrees with the previous reports. ${ }^{10}$ which is driven by the extended profile of potential at small ion valency.

\section{Conclusions}

In this study. the colloidal repulsion between two parallel cylindrical particles was investigated. The ligh counter ion valency of the electrolyte resulted in a fast decay of electrical potential. Subsequently. the middle point potential decay'ed rapidly, too. The lugher counter ion valency pronotes the 
screening of surface charge, so that the electrical potential decayed rapidly. On the while. the repulsive energy is increased with the valency at a very short separation distance. which is also observed in planar and spherical surfaces. Though Derjaguin approximation has a limit for its application, this approach provided an insight for the effect of counter ion valency of the electrolyte on the colloidal interaction. These results would be useful in fundamental study and engineering of many cylindrical colloidal particles such as a nanorod/nanotube suspension.

Acknowledgments. This work was supported by Korea Science and Engineering Foundation (Grant number: KOSEF M10755020001-08N5502-00110).

\section{References}

1. Caruso, F. Colloids and Collord Assemblies: Wiely-VCH: Weinheim, German, 2003; p 150

2. Niemeyer. C. M. Angew: Chem. Int. Ed. 2001, to. 4129.

3. van der Zande, B. M. I.; Bolnner, M. R.; Fokkink, L. G. I.: Schönenberger, C. Langmir 2000, 16,451 .

4. Dong, H.; Han, H.: Lee, S.-Y.J. Cryst. Growth 2008, 310, 1268.

5. Moon, J. H.; Kin, A. J.; Crocker, T. C : Yang, S. Adw Mater. 2007, 19, 2508 .

6. Moon, T.M.; Wei, A. J. Phns. Chem. B 2005, 109, 23336.

7. Dalv, B.: Arnold, D. C.; Kulkarni, J. S.; kazakova, O.; Shaw, M. T.: Nikitenko, S.: Erts, D.: Morris, M. A.; Holnes, J. D. Small 2006, 2, 1299

8. Lee, S.-Y.; Culver, J. N.; Harris, M. T. J. Coll Interface Sici. 2006, 297,554 .
9. Hunter, R. J. Folndations of Colloid Science; Oxford: New York, U.S.A., 1989: p 332.

10. Bowen, W. R.: Sharif, A. O. J. Colloid Interface Sci. 1997.187,363.

11. Sharif, A. O.; Tabatabaian, Z; Bowen, W. R. J. Colloid hnte face Sci. 2002, 255, 138

12. Halle, B. J. Chem. Phys 1995, 102, 7338

13. Choi, J.: Dong, H.: Haam, S.; Lee, S.-Y. Bull Korean Chem. Soc: $2008,29,1131$

14. Brenter, S. L.; McQuarrie, D. A. Biopinys. J. 1973, 13, 301.

15. Hanies, D. Langmuir $1998,14,3149$.

16. Ospeck, M.; Fraden, S. J. Chem. Phys. 1998, 109, 9166.

17. Tracy, C. A.: Widom, H. Phusica $41997,244,402$.

18. Brenter, S. L.; McQuarrie, D. A. J. Colloid Interface Sci. 1973, 4,298

19. James, E. A.; Williams, D. J. A. J. Colloid Interface Sci. 1985, 107,44

20. Bowen, W. R.; Sharif, A. O. Nature 1998, 398,663.

21. Chapot, D; Bocquet, L; Trizac, E. J. Colloid Interface Sci. 2005. 285.609

22. Chapot, D.: Bocquet, L:: Trizac, E. J. Chem. Phus 2005, 120, 3969.

23. Andrietti, F.; Peres, A.; Pezzotta, R. Biophws. J. 1976, 16, 1121

24. Hiemenz, P. C.; Rajagopalan, R. Principles of Colloid and Surface Chemistiv; Marcel Dekker: New York, U.S.A., 1997; p 502 .

25. Deggelmanı, M.; Graf, C.; Hagenbüchle, M.; Hoss, U.; Joluler, C.; Kramer, H.; Martin, C. Weber, R. I. Phws. Chem. 1994, 98, 364.

26. Israelachvili. I. Intemolecular \& Surface Forces: Academic Press: London, U.K., 1991: p 161.

27. Pack, G. R.: Wong L.: Lamm. G. Biopolwners 1999, $19,575$.

28. Gu. Y. J. Colloid Interface Sci. 2000, 231, 199.

29. Hsu, I.-P.: Yu, H.-Y.: Tseng, S. J. Phus. Chem. B 2006, 110. 25007.

30. Watanabe, A.: Sakamori, W. Colloid Polm . Sci. 1977, $255,782$. 